\title{
Improvement of an Efficient User Identification Scheme Based on ID-Based Cryptosystem
}

\author{
Eun-Jun Yoon and Kee-Young Yoo \\ Department of Computer Engineering, Kyungpook National University, \\ Daegu 702-701, Republic of Korea \\ Tel.: +82-53-950-5553, Fax: +82-53-957-4846 \\ ejyoon@infosec.knu.ac.kr, yook@knu.ac.kr
}

\begin{abstract}
In 2004, Hwang et al. proposed an efficient user identification scheme based on an ID-based cryptosystem that is suitable for the wireless/mobile environment. However, we find that their scheme is still vulnerable to impersonation attack. Accordingly, the current paper first shows the weakness of Hwang et al.'s scheme, and then presents an enhancement to resolve such problems.
\end{abstract}

Keyword: Mobile network, Security, Identity-based cryptosystem, User identification, Impersonation attack.

\section{Introduction}

In 1984, Shamir first proposed a user-identification scheme based on a public key cryptosystem that can let each user's identification information be his public key [1]. In this system, the public key of each entity is an identity which can be defined as that part of the identification information. In 1991, Maurer-Yacobi [2] proposed an identity-based non-interactive public key distribution system based on a novel trapdoor one-way function of exponentiation modulo of a composite number. In 1998, Tseng-Jan [3] modified a non-interactive public key distribution system and proposed several applications based on the Maurer-Yacobi scheme.

Recently, Hwang et al. 4] proposed an efficient scheme based on Tseng-Jan scheme that is more suitable for the wireless environment. Compared with TsengJan scheme, Hwang et al.'s scheme reduces the time required for responding and waiting. Additionally, their scheme uses the user's identity as his or her public key and does not need a key directory to store users' keys. However, we have found that their scheme is still vulnerable to impersonation attacks. Accordingly, the current paper first shows the weakness of Hwang et al.'s scheme, and then presents an enhancement to resolve such problems.

This paper is organized as follows: In Section 2, we briefly review Hwang et al.'s scheme. Section 3 shows how it suffer from vulnerability to impersonation attacks. In Section 4, we present an improvement of Hwang et al.'s scheme. In Section 5, we analyze the security of our proposed scheme. Finally, our conclusions are given in Section 6 .

\footnotetext{
* Corresponding author.
} 


\section{Review of Hwang et al.'s Scheme}

Hwang et al.'s scheme is composed of three phases: system initialization, user registration, and user identification.

\subsection{System Initialization Phase}

A trusted authority generates system parameters as follows:

- $M:$ the mobile device.

- $I D_{m}$ : the mobile device's identity.

$-B S:$ the base station.

- $I D_{b}$ : the base station's identity.

$-N$ : the product of four primes $p_{j}$, for $1 \leq j \leq 4$, whose decimal digits are between 60 and 70 and the numbers $\left(p_{j}-1\right) / 2$ are odd and pairwise relatively prime.

- e: a public integer in $Z_{\phi(N)}^{*}$.

$-d$ : a secret integer, which satisfies $e d \equiv 1(\bmod \phi(N))$.

- $t$ : a random number from $Z_{\phi(N)}^{*}$, where $\phi$ is the Euler's totient function.

$-s_{m}$ : a secret key of $M$, which satisfies $s_{m}=e \cdot t \cdot \log _{g}\left(I D_{m}^{2}\right) \bmod N$.

- $s_{b}$ : a secret key of $B S$, which satisfies $s_{m}=e \cdot t \cdot \log _{g}\left(I D_{b}^{2}\right) \bmod N$.

- $g$ : a primitive element in $G F\left(p_{j}\right)$.

$-h(\cdot)$ : a one-way function.

- $\|$ : a bits connection.

- T: a timestamp.

\subsection{User Registration Phase}

When mobile device $(M)$ joins the system, the procedures of user registration phase is shown as follows:

Step 1. $M$ presents his or her unique identity $I D_{m}$ to the trusted authority.

Step 2. The trusted authority computes $s_{m}=e \cdot t \cdot \log _{g}\left(I D_{m}^{2}\right) \bmod N, v=$ $t^{-1} \bmod \phi(N)$ and sends $s_{m}$ to $M$ as his or her secret key. Finally, the trusted authority publishes $\{N, g, e, h(\cdot)\}$ and keeps $\left\{p_{1}, p_{2}, p_{3}, p_{4}, t, v, d\right\}$ secret for all users.

Step 3. $M$ publishes $\left\{I D_{m}\right\}$ and keeps $\left\{s_{m}\right\}$ secretly.

\subsection{User Identification Phase}

Hwang et al. proposed a user identification scheme only using one pass to show the validity of a user's identity. Figure 1 shows Hwang et al.'s user identification phase. When the mobile device $(M)$ wants to show his identity $\left\{I D_{m}\right\}$ to the verifier the base station $(B S)$, the procedures of user identification phase is shown as follows: 
Mobile Device $(M)$

Base Station $(B S)$

Select $k \in Z_{N}^{*}$

$Y=\left(I D_{m}^{2}\right)^{k} \bmod N$

$Z=\left(I D_{b}^{2}\right)^{k \cdot s_{m} \cdot T} \bmod N$

$$
\stackrel{\left\{\left(I D_{m}\|Y\| Z\right), T\right\}}{\longrightarrow}
$$$$
Z^{\prime}=Y^{s_{b} \cdot T} \bmod N
$$$$
\text { Verify } Z \stackrel{?}{=} Z^{\prime}
$$

Fig. 1. Hwang et al.'s User Identification Phase

Step 1. $M$ chooses a random integer $k$ in $Z_{N}^{*}$ and computes $Y$ and $Z$ as follows:

$$
\begin{aligned}
& Y=\left(I D_{m}^{2}\right)^{k} \bmod N, \\
& Z=\left(I D_{b}^{2}\right)^{k \cdot s_{m} \cdot T} \bmod N .
\end{aligned}
$$

Then, $M$ sends $\left\{\left(I D_{m}\|Y\| Z\right), T\right\}$ to $B S$.

Step 2. After receiving the above messages from $M, B S$ computes $Z^{\prime}=Y^{s_{b} \cdot T}$ $\bmod N$, where $s_{b}\left(=e \cdot t \cdot \log _{g}\left(I D_{b}^{2}\right) \bmod N\right)$ is $B S '$ s secret key.

Step 3. $B S$ checks the equation $Z \stackrel{?}{=} Z^{\prime}$. If the equation holds, $B S$ will confirm that $M$ 's identity is valid.

\section{Impersonation Attack on Hwang et al.'s Scheme}

In Hwang et al.'s scheme, an attacker can easily impersonate a mobile device $(M)$. Suppose that the attacker has eavesdropped a valid message $\left\{\left(I D_{m}\|Y\| Z\right)\right.$, $T\}$ from the network. It is easy to obtain the message since they are exposed over the open network. Then the impersonation attack proceeds as follows:

Step $1^{*}$. The attacker select a timestamp $T^{*}$ and computes $Y^{*}=(Y)^{T} \bmod N$ and $Z^{*}=(Z)^{T^{*}}=\left(I D_{b}^{2}\right)^{k \cdot s_{m} \cdot T \cdot T^{*}} \bmod N$. Then, the attacker sends $\left\{\left(I D_{m}\left\|Y^{*}\right\| Z^{*}\right), T^{*}\right\}$ to the base station $(B S)$.

Step $2^{*}$. After receiving the attacker's message, $B S$ will compute $Z^{\prime}=\left(Y^{*}\right)^{s_{b} \cdot T^{*}}$ $\bmod N$ and check the equation $Z^{*} \stackrel{?}{=} Z^{\prime}$.

We can easily check that $B S$ will accept this message as follows:

$$
\begin{aligned}
Z^{*} & =\left(I D_{b}^{2}\right)^{k \cdot s_{m} \cdot T \cdot T^{*}} \bmod N \\
& =\left(g^{v \cdot s_{m} \cdot d}\right)^{k \cdot s_{b} \cdot T \cdot T^{*}} \bmod N \\
& =\left(I D_{m}^{2}\right)^{k \cdot s_{b} \cdot T \cdot T^{*}} \bmod N \\
& =\left(Y^{*}\right)^{s_{b} \cdot T^{*}} \bmod N \\
& =Z^{\prime}
\end{aligned}
$$


Attacker

Base Station $(B S)$

Eavesdrop $\left\{\left(I D_{m}|| Y \| Z\right), T\right\}$

Select $T^{*}$

$Y^{*}=(Y)^{T} \bmod N$

$Z^{*}=(Z)^{T^{*}}=\left(I D_{b}^{2}\right)^{k \cdot s_{m} \cdot T \cdot T^{*}} \bmod N$

$$
\underline{\left\{\left(I D_{m}\left\|Y^{*}\right\| Z^{*}\right), T^{*}\right\}}
$$$$
Z^{\prime}=\left(Y^{*}\right)^{s_{b} \cdot T^{*}} \bmod N
$$

Verify $Z^{*} \stackrel{?}{=} Z^{\prime}$

Fig. 2. Impersonation attack on Hwang et al.'s User Identification Scheme

In is thus apparent that Hwang et al.'s user identification scheme is insecure. Figure 2 shows an impersonation attack on Hwang et al.'s user identification scheme.

\section{Improvement of Hwang et al.'s Scheme}

In order to solve the weakness mentioned in Section 3, we propose an improved scheme. System initialization and the user registration phase are equal to Hwang et al.'s scheme. Figure 3 shows the proposed user identification phase. The procedures of proposed user identification phase are as follows:

Step 1. Mobile device $(M)$ chooses a random integer $k$ in $Z_{N}^{*}$ and computes $Y$, $Z$, and $C$ as follows;

$$
\begin{aligned}
Y & =\left(I D_{m}^{2}\right)^{k} \bmod N \\
Z & =\left(I D_{b}^{2}\right)^{k \cdot s_{m} \cdot T} \bmod N \\
C & =h(Z \| Y) .
\end{aligned}
$$

Mobile Device $(M)$

Base Station $(B S)$

Select $k \in Z_{N}^{*}$

$Y=\left(I D_{m}^{2}\right)^{k} \bmod N$

$Z=\left(I D_{b}^{2}\right)^{k \cdot s_{m} \cdot T} \bmod N$

$C=h(Z \| Y)$

$$
\underline{\left\{\left(I D_{m}\|Y\| C\right), T\right\}}
$$

$$
\begin{array}{r}
Z^{\prime}=Y^{s_{b} \cdot T} \bmod N \\
C^{\prime}=h\left(Z^{\prime} \| Y\right) \\
\text { Verify } C \stackrel{?}{=} C^{\prime}
\end{array}
$$

Fig. 3. Proposed User Identification Phase 
Then, $M$ sends $\left\{\left(I D_{m}\|Y\| C\right), T\right\}$ to the base $\operatorname{station}(B S)$.

Step 2. After receiving the above messages from $M, B S$ computes $Z^{\prime}$ and $C^{\prime}$ as follows;

$$
\begin{aligned}
& Z^{\prime}=Y^{s_{b} \cdot T} \bmod N, \\
& C^{\prime}=h\left(Z^{\prime} \| Y\right) .
\end{aligned}
$$

Step 3. $B S$ checks the equation $C \stackrel{?}{=} C^{\prime}$. If the equation holds, $B S$ will confirm that $M$ 's identity is valid.

\section{Security Analysis}

In this section, we shall only discuss the enhanced security features. The rest are the same as original Hwang et al.'s scheme as described in literature [4].

Definition 1. A secure one-way hash function is a function $f$ such that for each $x$ in the domain of $f$, it is easy to compute $f(x)$; but for essentially all $y$ in the range of $f$, it is computationally infeasible to find any $x$ such that $y=f(x)$ [5].

Theorem 1. In the proposed user identification phase, an attacker cannot impersonate a mobile device $(M)$.

Proof. An attacker can attempt to modify a message $\left\{\left(I D_{m}\|Y\| C\right), T\right\}$ into $\left\{\left(I D_{m}\left\|Y^{*}\right\| C^{*}\right), T^{*}\right\}$, where $T^{*}$ is the attacker's current timestamp. However, such a modification will fail in Step 3, because an attacker has no way of obtaining the valid value $Z=\left(I D_{b}^{2}\right)^{k \cdot s_{m} \cdot T} \bmod N$ to compute the valid parameter $C$. Furthermore, it is infeasible that an attacker can get $Z$ using $C=h(Z, Y)$, because it is a one-way property of a secure one-way hash function by Definition 1. Thus, the impersonation attack cannot be successful.

\section{Conclusion}

In this paper, we have demonstrated that Hwang et al.'s scheme is insecure against impersonation attacks. An improved version is then proposed to defeat impersonation attacks. The improved user identification scheme based on IDbased cryptosystem is designed to repair the security weakness inherent in Hwang et al.'s scheme.

\section{Acknowledgements}

We would like to thank the anonymous reviewers for their helpful comments in improving our manuscript. This research was supported by the MIC(Ministry of Information and Communication), Korea, under the ITRC(Information Technology Research Center) support program supervised by the IITA(Institute of Information Technology Assessment). 


\section{References}

1. Shamir, A.: Identity Based Cryptosystems \& Signature Schemes. Advances in Cryptology CRYPTO'84. Lecture Notes-Computer Science. (1984) 47-53

2. Maurer, U.M., Yacobi, Y.: A Non-interactive Public-key Distribution System. Designs, Codes and Cryptography. Vol. 9. No. 3. (1996) 305-316

3. Tseng, Y.M., Jan, J.K.: ID-based Cryptographic Schemes Using a Non-interactive Public-key Distribution System. Proceedings of the 14th Annual Computer Security Applications Conference (IEEE ACSAC98) Phoenix Arizona. (1998) 237-243

4. Hwang, M.S., Lo, J.W., Lin, S.C.: An Efficient User Identification Scheme Based on ID-based Cryptosystem. Computer Standards \& Interfaces. Vol. 26. No. 6. (2004) 565-569

5. Menezes, A.J., Oorschot, P.C., Vanstone, S.A.: Handbook of Applied Cryptograph. CRC Press. New York. (1997) 